\title{
Guidance on communicating about uncertainties in nuclear emergency management
}

\author{
T. Perko ${ }^{1, *}$, L. Benighaus ${ }^{2}$, Y. Tomkiv ${ }^{3}$ and H.V. Wolf ${ }^{4}$ \\ 1 SCK-CEN-Belgian Nuclear Research Centre, Mol, Belgium. \\ 2 DIALOGIK - non-profit institute for communication and cooperation research, Stuttgart, Germany. \\ ${ }^{3}$ NMBU/CERAD - Norwegian University of Life Sciences, Centre for Environmental Radioactivity, Ås, Norway. \\ ${ }^{4}$ UA - University of Antwerp, Antwerpen, Belgium.
}

\begin{abstract}
Within the European project CONFIDENCE, an extensive research programme has been conducted on a range of different tools, including Apps, SMS, numerical, narrative or mixed news messages and videos linked to uncertainty communication following potential nuclear or radiological emergencies. For this purpose, qualitative and quantitative research methods were applied in different European countries. Based on the results of these studies, we have formulated guidelines for efficient and effective communication about uncertainties that can be used in nuclear or radiological emergencies.
\end{abstract}

Keywords: uncertainties / communication / risk communication / nuclear emergency

\section{Introduction}

Communicating uncertainty requires that the facts relevant to recipients' decisions are identified, that the relevant uncertainties are characterized and their magnitude assessed, and that possible messages are drafted and their success evaluated (Fischhoff and Davis, 2014, p. 13671).

Uncertainty communication needs to be strategic, meaning that it should follow the objectives of emergency management and planning. It should be theory-based with respect to, for example, behaviour, information processing, social science, risk communication, and evidence-based in the sense that uses empirical data, surveys, observations or experiments. Moreover, the communication tools should be tested before largescale application. Communication about uncertainty should not be based on gut feelings and subjective opinions on "what may work" or what experts "would like to tell". This means that uncertainty communicators need to consider public perceptions, motivations, expectations and concerns, all of which are likely to differ from experts and from emergency to emergency. In addition, authorities and scientists (both natural and social) need to collaborate in order to communicate uncertainty successfully (Marignac et al., 2016).

Within CONFIDENCE, and as described in the following chapters, an extensive research programme has been conducted on a range of different communication tools, including Apps, short messages systems (SMS), various types of news

\footnotetext{
*Corresponding author: tanja.perko@sckcen.be
}

messages (e.g., numerical or narrative) and videos linked to uncertainty communication following potential nuclear or radiological emergencies. The methods applied a range of qualitative and quantitative approaches: workshops, round table discussions, public opinion surveys, observations, document analysis and case studies.

Based on the overall results of these studies, reported in detail in Perko et al. (2019a), we have formulated guidelines for efficient and effective communication about uncertainties.

\section{General suggestions on communicating uncertainty}

Addressing scientific and societal uncertainties in a nuclear emergency during pre-and-post radioactive release is not only an issue of decision-making, but also of public information and communication. Developing tools to deal with communication about uncertainties, either of technical or social nature, is crucial to improving protection, health and well-being of the affected population, and to enable informed decision making by the affected population as well as by experts. Communication strategies developed by different EU projects, as well as by risk communication researchers, for instance in European projects PREPARE, EAGLE (Perko et al., 2016b), CONCERT (Perko et al., 2019b), ARGOS, etc. highly advise to include information about uncertainties when communicating with the public, since it helps people to make informed decisions (Shirabe et al., 2015; Perko, 2016; Perko et al., 2016a). It is also advised that emergency actors admit uncertainties in 
communication to public(s) (IAEA, 2012, 2015; OECD/NEA, 2015; Jensen et al., 2017). However, systematic removal of uncertainty from public information is a common practice, especially in relation to emergency situations. Jensen et al. (2017) found that although scientists often try to thread uncertainty into their discourse (e.g. a limitations section), it has been observed that this information is systematically removed as scientific discovery is prepared for public communication (Jensen et al., 2017).

The FP7 project EAGLE revealed through discussions with experts that this systematic removal of uncertainty from public information related to ionising radiation is often done due to lack of methods and tools to communicate uncertainty information (http://eagle.sckcen.be/en/Deliverables).

In order to communicate efficiently and effectively during a nuclear or radiological emergency, the following general guidelines related to uncertainty communication are developed:

- decision-making involves uncertainty. Uncertainty in nuclear or radiological emergency management should be admitted and communicated. The more uncertain the information, the more communication is needed;

- be honest and open about what you do not know. Many words and expressions of common language can be used to express uncertainties. Instead of "uncertainty" the following words can be used: "there is considerable trust in ...", "many experts and scientists consider ...", "it is widely held that ...", "preliminary findings", "based on current measurements", "based on current insights", "as a first assessment", "further measurements are needed", "likely", "probably", "not certain", "may", "might", "seem"...;

- uncertainties can be reported in verbal, numeric, graphical or digital form. It is possible and advisable in some circumstances to use all of them simultaneously;

- information on the following types of uncertainties that present in nuclear or radiological management may be communicated: nature of uncertainties, magnitude of uncertainties, conflicting scientific evidence, scientific controversies, moral, societal, legal and other contextual uncertainties, statistical uncertainties, scenario uncertainties, level of theoretical understanding, level of empirical information (e.g., the empirical data that are lacking), quality of data, quality of model structure, limitations of methods and models, choices with respect to indicators, points of departure, important assumptions and practical limitations (e.g., the availability of iodine pills);

- when describing risks, be aware of the framing effects of wording, for example the use of the word "lives lost" versus "lives saved";

- when explaining protective measures use positive dominance, for example: "drink only bottled water" instead of "don't use tap water";

- aim for emergency management decisions that are robust with respect to the underlying uncertainties.

\section{How can Apps be used in communication about uncertainties?}

Information technology and apps meet many criteria for a successful communication tool during an emergency, as they rate high on effectiveness, efficiency, partly trust (depends on the topics and the source, API, 2016), and social acceptance. They are suitable for communicating with a broader population, except "hard-to-reach" target groups such as non-Internet users, the elderly population, or socially vulnerable groups (ethical aspects and uncertainty).

A non-systematic analysis of available communication tools reported in literature has been conducted. A literature search in the Web of Science focusing on methods, online tools, reports of projects and communication campaigns of how uncertainty was communicated in the event of nuclear emergency has been performed and resulted in 80 documents.

Although apps were supposed to be useful and credible, the problem may arise that participants feel too safe to use them (Reuter et al., 2017). Thus, crisis management via social media, although having a wide range and being easy and fast to distribute information, is not a simple tool when it comes to its effective use. There are risks of false information spreading, exclusion of specific groups and misunderstanding of the message (Stern, 2017, p. 11). Online communication additionally leads to less secondary crisis communication than conventional mediums (Utz et al., 2002, p. 45): people in directly affected areas wish to inform others about risks but have low effectiveness as they miss a useful informationtraceability system (Acar and Muraki, 2011, p. 399).

The following guides for Apps as communication tool are formulated:

- applying Apps for nuclear or radiological communication is nowadays inevitable;

- develop systems and Apps solely for the purpose of nuclear safety or integrate them into existing systems that are already used to inform and warn public about natural disasters like floods, fires and extreme weather conditions, after accidents in chemical factories, and attacks;

- carefully select which Apps to apply since there is a broad selection of existing technical tools, but the overall quality and the functionality of the different Apps vary greatly;

- given the great potential for a targeted use of various Apps for communicating uncertainty in the case of nuclear accidents, this topic should be further investigated;

- be aware of following issues related to Apps: false alerts, costs, labour demanding, tedious.

\section{How to formulate short text messages (SMS) for emergency warnings?}

Early warning systems by using mobile phones, sending short messages to people in the area under threat, are a potent component of public information in nuclear or radiological emergencies. In Europe, for instance, such early warning systems are used in Belgium (BE-alert) or in The Netherlands (NL-alert). The system is used for all types of emergencies and is not limited to radiological or nuclear emergencies.

CONFIDENCE partners conducted tests of short text messages (SMS) via workshops with lay people and students, as well as with experts and stakeholders in Spain, Greece, Sweden and at one CONFIDENCE training course (Duranova et al., 2020). In addition to this approach, SMS were tested during the ERPW2019 with conference 
participants (European Radiation Protection Week 2019), and during a training course with young researchers (Duranova et al., 2020).

Results show that SMS are accepted and used in everyday life, and people would be likely to accept them also as a means of warning via mobile phone. Comparing results from the various tests in different countries, people's opinions varied significantly about what would be an ideal SMS message that could be used for emergencies and warning. Noting the different languages and cultures in Europe, a one-size-fit-allsolution with a unique character of a SMS does not seem feasible.

As a general rule, however, a good SMS should be of medium length. A very short message reduces the message to a minimum, which could cause confusion and/or dread, a long one could be open to misinterpretation and a limited uptake of the information by the receiver. In the early phase of an emergency, communicating unclear or uncertain information like the extent of damage and release of radioactivity is recognised, but needs frequent updates (like sending several messages) and additional communication means. SMS should be designed individually and tailored for each country and also each nuclear power plant.

Further in-depth studies specifically on the effect of SMS in a stressful situation like an emergency are advised, with a larger group of research participants. The findings from conferences and cases from Spain, Sweden, Greece and the CONFIDENCE training courses highlight the need to continue testing information messages for nuclear emergency communication with experimental methods, in order to be better prepared for a future accident. Both the content of the messages and the preferred channels (SMS, WhatsApp...) should be further investigated. The tests with SMS messaging also show that it is necessary to tailor messages to both experts and the general population, as it would be assumed that relevant differences would exist between them.

The following guidelines are formulated related to the SMS as a warning in case of a nuclear or radiological emergency:

- inform or warn local residents and general population by using SMS (short text messages) is a rather new and effective communication practice among early notification systems. People nowadays use mobile telephones and smart phones constantly, thus this type of system could effectively warn people about the ongoing emergency;

- the SMS should be strategically predesigned and methodologically tested before the application. Specific attention should be paid to the information needs of residents and their understanding of the message;

- send the SMS in local language but also in English language;

- the optimal length of the SMS is approximately 200 characters, but the specificity of the local language should be taken into account;

- in the early phase of an emergency, there will be a need to communicate uncertain information like extent of damage and release of radioactivity. Therefore, frequent updates (like sending several messages) on the situation will be required and hyperlinks to additional communication means should be included;
- content of the SMS should warn people, (e.g. "Warning!"), clearly indicate what happened (e.g. "Accident at $\mathrm{ABC}$ Nuclear power plan") and where (e.g. "in place, country"). Uncertainty should be expressed (e.g. "Release of radioactivity not yet confirmed") and the message should tell people what to do (e.g. "Stay inside, close windows and shut down ventilation system"). People should be informed about where they can get more information (e.g. "Listen to local news, check updates at hyperlink"). The name of the organization behind the SMS should be included in the message, e.g. "Federal crisis centre";

- SMS should be designed for each country and each nuclear power plant (e.g. name of the NPP).

\section{How to address uncertainty in waiting rooms}

One of the main lessons learned in the two days long measurement campaign after a radiological emergency in Fleurus, Belgium (2008) was that more attention should be paid to communication in the waiting rooms (Perko et al., 2014). Waiting rooms proved to be a bottleneck of the emergency. People, mainly pregnant women, young parents, children were waiting for hours to be measured. Most of them didn't know what radioactive iodine was, what they will be measured for, by whom and how. The atmosphere in the waiting room was stressful and gave the affected population feelings of uncertainty (Perko et al., 2014). The following questions from local residents were reoccurring:

- What and how are experts going to measure? How to explain that the expert is going to measure the possible existing radioactivity in the body and will not be exposing the measured person to radiation?

- What does mSv mean? How to communicate with pregnant women in order to address their concerns? How to explain the results of the measurements? (most people don't know that body itself contains some radioactivity);

- What is iodine, radio-iodine or caesium 137? What are iodine tablets and why shouldn't one take them immediately?

After the event, the experts providing the measurement concluded that communication should be prepared in advance and should be a part of the overall measurement strategy. Therefore, they suggested to actively provide information about topics like health effects of radiation and radiation measurement principles. This information material should focus on various target audiences (children, pregnant women, teachers and general public) and be distributed already in a waiting room (Van der Meer et al., 2010).

The following guides related to the communication in waiting rooms are formulated:

- the standard information that has to be communicated in waiting rooms (decontamination center, emergency rooms, measurement room, etc.) includes the following: what is radiation and what is contamination; health symptoms of radiation; description of the decontamination process; radionuclides e.g. ${ }^{131} \mathrm{I},{ }^{137} \mathrm{Cs},{ }^{136} \mathrm{Cs},{ }^{60} \mathrm{Co}$ for the different 
types of accidents; explanation on how internal or external contamination is measured; identification and the expertise of institution that is providing measurement service; efficacy of processes, for instance decontamination;

- the information material should be designed in a way that people in a stressful situation can process it: that they can understand, comprehend, and remember the information;

- use simple terms and explanations (e.g. "Contamination is like being wet from the rain and irradiation is like sunbathing"); formulate positive statements (e.g. "Drink only bottled water" instead of "Don't drink tap water"); address psychometric risk characteristics (e.g.: familiarity "Germanium detector measures potential radiation on the same way as thermometer measures body temperature", dread characteristic, e.g. break down the process in different steps e.g. First you will do this, then you go there, followed by..."; effect on children e.g. use images of children at the communication material);

- communication tools in waiting rooms should be in diverse formats: leaflets, posters, videos, and other multimedia. Face to face communication is the most efficient, but also the most resource-consuming;

- create video material, which can inform people in the waiting room about how measurements will be done and what will be measured in the next room and by whom these measurements will be performed.

\section{News Media: the effectiveness of numerical and narrative messages}

The news media continue to be one of the most powerful tools to reach a broad public, be it via television, radio or online and print news. Preparatory risk communication and postemergency recovery communication should make use of these channels to ensure that a broad public is reached, also including, for instance, the elderly population. Research in the domains of persuasive communication and evidence effectiveness tells us that different types of news messages may result in considerably different perceptual and behavioral responses among the recipients (Reynolds and Reynolds, 2002, p. 429), which is why uncertainty communication should consciously consider which type of message will be most suitable for the respective communication. Research postulates that whenever a claim is made that aims to persuade the public of behaving in a certain way, for instance when an immediate behaviour is required in an emergency, "evidence" is needed to substantiate this claim for it to be considered valid by the recipient and to advocate a change in his or her behaviour (Wojcieszak and Kim, 2016). Such a change in behaviour may be about consuming food products, in the case of food risk communication, or ensuring that the public understand the importance of waiting with the uptake of iodine tablets in case of a radiological emergency. Evidence may for instance be provided in the form of numbers that "prove" a claim, as well as in a more narrative manner, for instance through personal testimonies. Sound risk communication drives people's attention, is well understood by lay people, is recalled when needed and, most importantly, is accepted by people leading them to act in accordance with the advised behaviour in case of an emergency. Unfortunately, experience from different emergency responses and studies show that sound communication about radiological risks is difficult to achieve.

Within a representative face-to-face survey of the Belgian population $(N=1086)$, conducted over a 3-month-period from December 2017 to February 2018, the authors ran two parallel experiments: the French-speaking language group was exposed to a pre-emergency news article manipulation targeting uncertainties related to waiting for instructions for the uptake of iodine pills, the Dutch-speaking group to a postemergency news article manipulation targeting uncertainties related to the safe consumption of food from Fukushima. The two experiments consisted of 3 experimental conditions each ( 1 numerical message, 1 narrative message (personal testimony), 1 combined message); control questions were included in the respective other group. Each participant read one news article containing a manipulated claim and answered post-test questions regarding their recall and acceptance of the message, their message ratings, and their behavioural intentions regarding the uncertainty communicated. In addition, the authors tested whether the effectiveness of different messages differs for people with different predispositions (e.g. sociodemographics, preference for numbers, empathic score). This study investigated what kind of communication is most effective and what kind of communication achieves better response by a population in a radiological emergency.

Results revealed that for post-emergency communication on the consumption of food from Fukushima, numerical messages were perceived as more effective than narrative or combined messages, and that numerical messages led to higher message acceptance than both other conditions. Prior attitudes towards nuclear energy as well as the respondents' trust in authorities proved to be key control variables, with those holding more negative attitudes being more critical of the message. In addition, all three messages types lowered the perceived risk from the issue in question when compared to the control group, which shows that communication about uncertainties in general is effective in reducing inhibitions.

The following guidelines related to the communication about food products are formulated:

- provide precise unambiguous numerical data to support your message to improve the perceived credibility of your claim. Few and easily understandable numbers may present an apt strategy to induce positive behavioural changes;

- target different subgroups of the public with varying frequency and message types to increase overall trust levels. If issue attitudes among a specific population group can be evaluated, those consumers who are inclined to be critical towards the given issue may need more frequent and more diverse messages in order to accept a message and change their behaviour accordingly. A fruitful pathway may be to communicate a variety of messages that include different types of numbers, but also testimonies by experts and ordinary citizens, in order to provide a nonauthoritative picture that those low on trust do not distrust a priori;

- besides the communication of the uncertainty, efforts aimed at enhancing the public's overall trust in authorities should be undertaken. A significant fraction of the population has entered a spiral of distrust in authorities, 
which may lead to long-term problems for emergency communication as low levels of trust may inhibit the consumers' readiness to accept communicated messages. Considering to also approach alternative news websites may be a first path to tackle this problem for risk communicators;

- provide numbers if the message to be communicated contains a threat that is considered distant rather than imminent, for example risk communication that aims to prepare for nuclear emergencies. If a threat is imminent, such as an acute food risk in the region, we suggest to broaden the scope of messages by also providing more emotionallyinvolving messages, such as personal testimonies.

Results for the iodine pills are reported in Turcanu et al. (2020).

\section{Conclusions}

In this paper, we described developments and tests of communications tools performed within the CONFIDENCE project. The results showed that there is a great potential in the various tools that would allow for better communication of uncertainty in an emergency situation.

In order to improve further communication about uncertainty, the authors suggest developing a large scale demonstration project where these tools could be tested in different emergency management situations and different cultural and mass media environments. Moreover, additional research is needed to understand the influence of uncertainty communication on risk perception, trust and behaviour and how exchange or sharing of uncertainty information between and among different parties (such as regulators, experts, consumers, media, general public) can be provided.

Acknowledgement. The work described in this paper was conducted within the CONFIDENCE project which was part of the CONCERT project. This project has received funding from the Euratom research and training programme 20142018 under grant agreement No. 662287. This work was also partly supported by the Research Council of Norway (RCN) (Grant Number 223268/F50 [CERAD - Centre for Environmental Radioactivity] and Grant Number 263856/E40).

Disclaimer (Art. 29.5 GA). This publication reflects only the author's view. Responsibility for the information and views expressed therein lies entirely with the authors. The European Commission is not responsible for any use that may be made of the information it contains.

\section{References}

Acar A, Muraki Y. 2011. Twitter for crisis communication: Lessons learned from Japan's tsunami disaster. Int. J. Web Based Communities 7(3): 392-402.

American Press Institute and the Associated Press-NORC Center for Public Affairs Research (API). 2016. A new understanding. What makes people trust and rely on news. Media Insight Project. Available from https://www.americanpressinstitute.org/publica tions/reports/survey-research/trust-news/single-page/.
Duranova T, Beresford NA, Perko T, Raskob W. 2020. Education and training activities in the Euratom CONFIDENCE project. Radioprotection 55(HS1). https://doi.org/10.1051/radiopro/ 2020011.

Fischhoff B, Davis LA. 2014. Communicating scientific uncertainty. PNASS 111: 13664-13671.

IAEA. 2012. Communication with the public in a nuclear or radiological emergency. EPR-Public Communications. Vienna: IAEA.

IAEA. 2015. Method for developing a communication strategy and plan for a nuclear or radiological emergency, emergency preparedness and response. EPR Public Communication Plan. Vienna, Austria: International Atomic Energy Agency, p. 23.

Jensen JD, Pokharel M, Scherr CL, King AJ, Brown N, Jones C. 2017. Communicating uncertain science to the public: How amount and source of uncertainty impact fatalism, backlash, and overload. Risk Anal. 37: 40-51.

Marignac Y, Hazemann J, Baudé SJR. 2016. Managing the complexity of societal needs in a nuclear emergency situation: Towards further experts collaboration for the "enlightened protection" of populations. Radioprotection 51: S159-S161.

OECD/NEA. 2015. Stakeholder involvement in decision making: A short guide to issues, approaches and resources. Paris, France: OECD.

Perko T. 2016. Risk Communication in the case of the Fukushima accident: Impact of communication and lessons to be learned. Integr. Environ. Assess. Manag. 12: 683-686.

Perko T, Thijssen P, Turcanu C, Van Gorp B. 2014. Insights into the reception and acceptance of risk messages: Nuclear emergency communication. J. Risk Res. 17: 1207-1232.

Perko T, Tomkiv Y, Prezelj I, Cantone MC, Gallego E, Oughton DH. 2016a. Communication with media in nuclear or radiological emergencies: General and practical recommendations for improvement. J. Radiol. Prot. 51(HS2): S163-S169.

Perko T, Zeleznik N, Mays C, Diaconu D, Kralj M, Koron B. 2016 b. Communication recommendation related to ionising radiation, EAGLE, Enhancing educAtion, traininG and communication processes for informed behaviors and decision-making reLatEd to ionizing radiation risks. Brussels, Belgium: EC.

Perko T, Benighaus L, Tafili V, Oughton DH, Tomkiv Y, Sala R, Germán S, López S, Oltra C, Duranova T, Raskob W, Müller T, Nishizawa M, Wolf HV, Thijssen P, Camps J, Turcanu C, Benighaus C, Moschner J, Renn O. 2019a. Guidelines on tools for communication of uncertainties. CONCERT Deliverable D9.29. Available from https://www.concert-h2020.eu/en/Publications.

Perko T et al. 2019b. Towards a strategic research agenda for social sciences and humanities in radiological protection. J. Radiol. Prot. 39: 766-784.

Reuter C, Kaufhold MA, Leopold I, Knipp H. 2017. KATWARN; NINA or FEMA? Multi-method study on distribution, use, and public views on crisis Apps. In: 25th Eur. Conf. on Information Systems (ECIS), Guimaraes, Portugal. Available from http://www. peasec.de/paper/2017/2017_ReuterKaufholdLeopoldKnipp_Crisi sApps_ECIS.pdf.

Shirabe M, Fassert C, Hasegawa R. 2015. From risk communication to participatory radiation risk assessment. Fukushima Glob. Commun. Progr. Work. Pap. Ser. 21: 2-8.

Stern E. 2017. Unpacking and exploring the relationship between crisis management and social media in the era of "smart devices". Homel. Secur. Aff. 13, Article 4. Available from https://www.hsaj. org/articles/13986.

Turcanu C, Perko T, Sala R, Wolf HV, Camps J, Oughton DH. 2020. Social uncertainties related to stable iodine intake in a nuclear 
emergency. Radioprotection. https://doi.org/10.1051/radiopro/ 2020027.

Utz S, Schultz F, Glocka S. 2002. Crisis communication online: How medium, crisis type and emotions affected public reactions in the Fukushima Daiichi nuclear disaster. Public Relat. Rev. 39(2013): 40-46.

Van der Meer K et al. 2010. Thyroid measurement campaign after an accidental release of $50 \mathrm{GBq} 131 \mathrm{I}$ in Fleurus, Belgium. In:
Third European IRPA Congress on Radiation Protection, Helsinki.

Reynolds RA, Reynolds JL. 2002. Evidence. In: The SAGE hand book of persuasion: Developments in theory and practice (J.P. Dillard, M. Pfau, Eds.), pp. 427-444. Thousand Oaks, CA: Sage.

Wojcieszak M, Kim N. 2016. How to improve attitudes toward disliked groups. Commun. Res. 43(6): 785-809. Available from https://doi.org/10.1177/0093650215618480.

Cite this article as: Perko T, Benighaus L, Tomkiv Y, Wolf HV. 2020. Guidance on communicating about uncertainties in nuclear emergency management. Radioprotection 55(HS1): S169-S174 